\title{
PACIENTES VIVENDO COM HIV/AIDS E COINFECÇÃO TUBERCULOSE: DIFICULDADES ASSOCIADAS À ADESÃO OU AO ABANDONO DO TRATAMENTO
}

\author{
Manoel Pereira de Sousa FILHO ${ }^{a}$, Izaildo Tavares LUNA ${ }^{b}$, \\ Kelanne Lima da SILVA ${ }^{c}$, Patrícia Neyva da Costa PINHEIRO ${ }^{\mathrm{d}}$
}

\section{RESUMO}

Pesquisa realizada em Fortaleza-CE, entre março e abril de 2011, com o objetivo de identificar as dificuldades que influenciam a adesão ou o abandono do tratamento de tuberculose em pacientes com Síndrome da Imunodeficiência Adquirida. Estudo qualitativo, com informações coletadas por meio de entrevista semiestruturada aplicada a pacientes com Síndrome da Imunodeficiência Adquirida e coinfecção tuberculose. Utilizamos o discurso do sujeito coletivo para a análise dos resultados. Os pacientes relataram dificuldades relacionadas aos aspectos socioeconômicos, ao estilo de vida e ao uso da medicação. O uso de álcool e o consumo de drogas ilícitas surgiram como fatores que levam a episódios de interrupção do processo terapêutico da doença. Concluímos que as barreiras relacionadas aos aspectos sociais, econômicos e ao estilo de vida são mais difíceis de serem enfrentadas para uma adesão continuada ao tratamento, tornando necessário o papel desempenhado pelos profissionais da saúde, apoiado por políticas públicas e sociais mais resolutivas.

Descritores: Síndrome da Imunodeficiência Adquirida. Tuberculose. Adesão à medicação.

\section{RESUMEN}

Investigación realizada en Fortaleza-CE, entre marzo y abril de 201 1, con el objetivo de describir las dificultades que influyen en la adhesión o abandono del tratamiento de la tuberculosis en pacientes infectados con Virus de la Inmunodeficiencia Humana. Estudio cualitativo, con informaciones recopiladas a través de entrevista semiestructurada, aplicada en pacientes con Síndrome de Inmunodeficiencia Adquirida coinfectados con tuberculosis. Se utilizó el Discurso del Sujeto Colectivo para el análisis de los resultados. Los pacientes relataron dificultades cuanto a los aspectos socioeconómicos, estilo de vida y uso de medicamentos. El uso de alcohol y consumo de drogas fueron factores que llevaron a episodios de interrupción del proceso terapéutico de la enfermedad. Concluimos que obstáculos relacionados a los aspectos sociales, económicos y estilo de vida son más difïciles de enfrentar para una adhesión continuada al tratamiento, se hace necesario el rol desempeñado por profesionales de salud, con el apoyo de políticas sociales más resolutivas.

Descriptores: Síndrome de Inmunodeficiencia Adquirida. Tuberculosis. Cumplimiento de la medicación Título: Pacientes que viven con el VIH/SIDA y coinfectados con tuberculosis: las dificultades asociadas con la adhesión o abandono del tratamiento.

\section{ABSTRACT}

Research carried out in Fortaleza-CE, between March and April 2011, with the objective of describing the difficulties that influence non-compliance to tuberculosis treatment or treatment dropout in patients infected with Human Immunodeficiency Virus. Qualitative study with data collected through a semi-structured interview, applied to patients with acquired immunodeficiency syndrome and with co-infection of tuberculosis. The Collective Subject's Speech was used for result analysis. Patients reported difficulties related to socioeconomic aspects, lifestyle and drug use. The consumption of alcohol and illicit drugs were factors that led to the discontinuance of the therapeutic process of the disease. We conclude that the barriers related to social economical aspects and to lifestyle are more difficult to be faced for a continuous compliance to treatment, making the role played by health professionals necessary, supported by more effective public and social policies.

Descriptors: Acquired Immunodeficiency Syndrome. Tuberculosis. Medication compliance.

Title: Patients living with HIV/AIDS and co-infection by tuberculosis: difficulties associated with treatment compliance or dropout.

a Enfermeiro do Hospital São José, especializado em Doenças Infecciosas. Fortaleza, Ceará, Brasil.

b Enfermeiro, Mestrando do Programa de Pós-Graduação em Enfermagem da Universidade Federal do Ceará (UFC), Professor Substituto do Departamento de Enfermagem da UFC, Especialista em Vigilância Epidemiológica das Doenças Transmissíveis, Bolsista Capes. Fortaleza, Ceará, Brasil.

c Enfermeira, Mestranda do Programa de Pós-Graduação em Enfermagem da UFC, Especialista em Saúde da Família. Fortaleza, Ceará, Brasil.

d Enfermeira, Doutora em Enfermagem, Professora Adjunta do Departamento de Enfermagem da UFC. Fortaleza, Ceará, Brasil. 


\section{INTRODUÇÃO}

A tuberculose (TB) é uma das principais causas de adoecimento e morte no mundo, principalmente na África e na Ásia. Em 2006, ocorreram 9 milhões de novos casos de tuberculose, dos quais 700 mil foram em pacientes co-infectados. Dos 1,7 milhões de mortes por tuberculose que aconteceram nesse mesmo ano, 200 mil foram pacientes com infecção pelo HIV. ${ }^{(1)}$

No mundo, atualmente, cerca de dois bilhões de pessoas estão infectadas pela tuberculose pulmonar, a maioria residindo nos países em desenvolvimento. Nesses países a OMS registra oito milhões de novos casos de tuberculose-doença por ano e, destes, cerca de três milhões vão a óbito, ou seja, há 35\% de letalidade, número bem maior que a média prevista com o advento da quimioterapia tuberculostática ${ }^{(2)}$.

Desde o início da epidemia da AIDS ou SIDA (Acquired Immunodeficiency Syndrome - Síndrome de Imunodeficiência Adquirida) até 2009, no mundo mais de 60 milhões de pessoas foram infectadas pelo HIV, e quase 30 milhões de pessoas morreram de causas relacionadas ao HIV. Um em cada quatro óbitos por AIDS é causado por tuberculose, uma doença que pode ser prevenida e curada ${ }^{(3)}$.

No Brasil, cerca de 40 milhões foram infectados com o M. Tuberculosis (25\% da população), com aproximadamente 100 mil casos novos de tuberculose-doença por ano, dos quais de quatro a cinco mil vão a óbito, uma letalidade em torno de $5 \%$. A incidência de tuberculose nos pacientes infectados pelo Vírus da Imunodeficiência Humana é de 8\% a 10\% ao ano. Cerca de $37 \%$ das pessoas soropositivas infectadas pelo $M$. tuberculosis desenvolvem tuberculose nos seis meses seguintes à infecção e, destes, 2\% a 5\% desenvolvem a doença nos dois anos seguintes à infecção ${ }^{(4)}$.

Vale destacar que o Brasil passou da $16^{\mathrm{a}}$ para a $17^{\mathrm{a}}$ posição no ranking dos 22 países com maior registro de casos de tuberculose no mundo. No entanto, o índice de casos aumentou de 39 para 48 por 100 mil habitantes, de acordo com o Relatório de Controle Global da Tuberculose 2009, lançado pela Organização Mundial de Saúde de $2009^{(5)}$.

Na distribuição de casos novos de tuberculose por unidade federada observa-se que os Estados do Rio de Janeiro, Amazonas, Pernambuco, Pará, Rio Grande do Sul, Bahia, Ceará, Acre, Alagoas e Maranhão possuem taxas de incidência superiores a 38,2 casos por 100.000 habitantes. Em 2007, Fortaleza, a
Capital do Estado do Ceará, apresentou-se como a cidade brasileira com maior incidência de TB, com 66,3 casos novos/100.000 habitantes ${ }^{(6)}$.

Em relação à situação epidemiológica da tuberculose no Ceará, no ano de 2009 foram notificados pelo Sistema de Informação de Agravos de Notificação (SINAN), 3.168 novos casos de tuberculose, correspondendo a um coeficiente de incidência de 37,1 por 100 mil habitantes, índice que ficou abaixo do registrado em 2008, quando ocorreram 3.721 novos casos, ou seja, 44,0 por 100 mil habitantes. Destes, $70 \%$ apresentaram cura, $7,6 \%$ abandonaram o tratamento, e houve uma taxa de mortalidade de 3,1/100000hab. Dos casos notificados de tuberculose no Ceará, em 2009, 1.675 eram pulmonares bacilíferos, sendo um percentual de $55 \%$ de pacientes que alcançam essas formas. Isto significa que a transmissão está ativa e os pacientes estão sendo diagnosticados e tratados tardiamente ${ }^{(7)}$.

Em nosso País, a tuberculose constitui uma das co-infecções mais frequentes que conduzem ao óbito pessoas infectadas pelo Vírus da Imunodeficiência Humana (HIV). A duração mínima do tratamento de tuberculose é de seis meses, período em que muitas pessoas abandonam ou fazem uso irregular dos tuberculostáticos e/ou dos antirretrovirais, o que favorece o desenvolvimento da resistência ${ }^{(8)}$.

Apesar de existirem recursos tecnológicos capazes de promover o controle da disseminação da tuberculose, ainda não há perspectivas de se obter, em futuro próximo, a sua erradicação, a não ser que novas vacinas ou tratamentos sejam descobertos. Além disso, a associação da tuberculose com a AIDS representa um novo desafio em escala mundial devido à alta taxa de mortalidade ${ }^{(9)}$.

Observamos que a tuberculose pulmonar em pessoas infectadas pelo HIV continua sendo um problema relevante para a saúde pública do Brasil. Os desafios atuais das políticas públicas são facilitar a adesão ao tratamento, promover informações clínicas, melhorar as condições socioeconômicas e educacionais, envolver familiares e profissionais de saúde no processo e oferecer assistência integral ao paciente com co-infecção tuberculose/AIDS, visando à adesão e ao não-abandono do tratamento.

Para tanto, a equipe de saúde precisa compreender a multiplicidade de fatores condicionantes à adesão ao tratamento da tuberculose pelos pacientes, buscando, assim, envolver os vários níveis de complexidade do sistema de saúde necessários para o desenvolvimento e implementação de ações de 
impacto que possam vir a interferir na adesão, sendo imperativo o reconhecimento das especificidades da sua clientela em particular.

Diante do exposto, emergiu o seguinte questionamento: quais as dificuldades que contribuem para o abandono ou a adesão ao tratamento de TB na visão da pessoa infectada pelo HIV?

Acreditamos que as respostas a este questionamento poderão contribuir com dados subjetivos para a compreensão do processo de não-adesão e abandono ao tratamento, fornecendo ao profissional de saúde uma visão ampliada dos casos de recidiva da tuberculose no portador do HIV/AIDS, proporcionando o planejamento de intervenções preventivas visando às individualidades e dificuldades de cada pessoa.

O objetivo deste estudo é identificar as dificuldades associadas à adesão ou ao abandono ao tratamento da tuberculose em pacientes vivendo com HIV/AIDS em terapia antirretroviral altamente ativa.

\section{METODOLOGIA}

Estudo do tipo descritivo e exploratório, com abordagem qualitativa, pois envolve a compreensão acerca dos acontecimentos, das vivências únicas dos sujeitos, das influências culturais e das percepções construídas ao longo da vida ${ }^{(10)}$.

Foi realizado em um hospital de referência em doenças infecciosas, órgão com personalidade jurídica de direito público, vinculado à Secretaria da Saúde do Estado do Ceará, localizado na cidade de Fortaleza. Os sujeitos foram sete pacientes com diagnóstico médico confirmado de HIV com co-infecção tuberculose/AIDS.

Para a seleção dos participantes utilizamos os critérios de inclusão: ter diagnóstico confirmado de HIV com co-infecção tuberculose/AIDS constatado no prontuário; ser maior de 18 anos; residir em Fortaleza-CE; estar em tratamento atual de TB com uso domiciliar de medicamentos e em uso de antirretroviral; e ter tido, no mínimo, um abandono anterior confirmado de tratamento de tuberculose.

O estudo foi submetido ao Comitê de Ética do referido hospital, conforme Resolução 196/96, do Conselho Nacional de Saúde, do Ministério da Saúde, e aprovado sob protocolo nº. 058/2009.

Os sujeitos do estudo foram esclarecidos sobre o objetivo da pesquisa, sendo garantido o anonimato às informações, além do direito de desistir em qualquer momento de participar da pesquisa, em qualquer uma de suas etapas. Foi apresentado, para a assinatura de todos os participantes, o Termo de Consentimento Livre e Esclarecido.

As informações foram coletadas em março e abril de 2010, por meio de entrevista orientada por perguntas relacionadas aos objetivos deste estudo, sendo três questões tratadas neste artigo: como se sente realizando o tratamento? Quais as dificuldades enfrentadas ao realizar o tratamento? Quais os motivos que o levaram a desistir do tratamento terapêutico?

Para a organização e análise das informações utilizamos a técnica do Discurso do Sujeito Coletivo (DSC), que trata de uma "estratégia metodológica com vista a tornar mais clara uma dada representação social e o conjunto dos aspectos que confirma um dado imaginário” (11).

As entrevistas foram gravadas, transcritas, digitadas e ouvidas exaustivamente para confirmação da fidedignidade das falas. A leitura dos depoimentos foi realizada repetidamente, para codificação e identificação das unidades significativas, destacando expressões-chave e ideias centrais semelhantes ou complementares em um discurso-síntese, que consiste no Discurso do Sujeito Coletivo (DSC), discutido e analisado à luz da literatura concernente ao assunto.

\section{RESULTADOS E DISCUSSÕES}

Em meio ao atendimento realizado aos pacientes que abandonaram o tratamento para tuberculose, junto aos critérios de seleção do estudo foi possível conhecer os sete sujeitos envolvidos nesta pesquisa, sendo todos do sexo masculino, na faixa etária entre 30 e 40 anos. Quanto ao perfil ocupacional, dois são serventes, um é vigilante, dois são cozinheiros e dois estão desempregados.

Ainda com relação ao perfil desses sujeitos, no que concerne à escolaridade, três têm o Ensino Fundamental incompleto, um tem o Ensino Médio incompleto e três, o Ensino Médio completo. Em relação ao tempo de diagnóstico da AIDS, os sete pacientes descobriram que estavam com a doença há menos de seis anos. Quanto ao tempo de diagnóstico da co-infecção tuberculose/AIDS, três tiveram o diagnóstico entre os anos de 2007 e 2009, e quatro tiveram o diagnóstico em 2010.

As informações das entrevistas geraram seis Ideias Centrais (ICs), sendo duas para cada questão norteadora: boa aceitação ao tratamento; dificuldades 
em realizar o tratamento; dificuldades relacionadas ao paciente e ao estilo de vida; dificuldades com os regimes terapêuticos e confusão sobre os intervalos entre as doses; consumo de drogas ilícitas como barreira à adesão contínua ao tratamento; uso recreacional do álcool como fator de interrupção da tomada do remédio.

Ideia Central 1: Boa aceitação ao tratamento:

[...] me sinto bem graças aos remédios e graças ao hospital que tá tendo e pode fornecer pra gente. Então eu posso me tratar, no início sentia muito mal, mais fora isso eu me trato aqui no hospital desde o ano passado e foi até hoje, fiquei muito bem [...].

Percebemos que os entrevistados relataram que se sentem muito bem em poder realizar o tratamento, apesar de terem apresentado algumas dificuldades no início, em virtude dos efeitos colaterais da medicação.

Conforme as diretrizes preconizadas pelo $\mathrm{Mi}-$ nistério da Saúde, a adesão adequada aos esquemas antituberculose e Antirretroviral (ARV) concomitante é um grande desafio para os pacientes, pois os elevados números de comprimidos e cápsulas a serem ingeridos e a ocorrência dos efeitos colaterais acarretam no paciente o adiamento do $\mathrm{ARV}^{(12)}$.

Durante o tratamento antituberculose e antirretroviral recomenda-se que o paciente receba supervisão periódica na autoadministração das doses, intensificando as medidas profiláticas e preventivas, o vínculo com o serviço de saúde, o entendimento do tratamento e dos efeitos colaterais possíveis para que a adesão terapêutica ocorra sem episódio de abandono ${ }^{(13)}$.

Ideia Central 2: Dificuldades em realizar o tratamento:

[...] eu me sinto assim com um pouco de dificuldade pra seguir com o tratamento, porque às vezes dar diarréia, falta apoio, falta dinheiro para o transporte. Essas são algumas dificuldades que agente tem pra poder conseguir realizar este tratamento [...].

As dificuldades que influenciam a não-adesão e o abandono do tratamento de TB em pessoas vivendo com HIV/AIDS incluem o baixo nível educacional e socioeconômico, os hábitos de vida prejudiciais à saúde, a falta de recursos para alimentação e locomoção, o uso de álcool e outras drogas, a história de não-adesão anterior, os efeitos adversos da me- dicação, a não-aceitação do diagnóstico, a melhora dos sintomas e a ausência de conhecimento sobre a evolução clínica e importância do tratamento ${ }^{(14)}$.

A adesão à terapêutica é um fenômeno sujeito à influência de múltiplos fatores que afetam diretamente o paciente. Estes determinam o comportamento da pessoa em relação às recomendações referentes ao tratamento da doença. Esses diferentes fatores estão relacionados às condições socioeconômicas, à doença, à terapêutica, à relação dos profissionais de saúde com o paciente, bem como ao próprio paciente ${ }^{(15)}$.

Quanto aos fatores sociais e econômicos, estes podem interferir na adesão ao tratamento de acordo com a dificuldade que o paciente tem para conseguir adquirir o medicamento, seja pelo preço, no caso da necessidade de compra, seja pela ausência do medicamento na unidade de saúde ou na farmácia comercial; pela falta de dinheiro para a locomoção até a unidade de saúde onde realiza o tratamento, como também pela distância entre a unidade de saúde ou a farmácia e a residência do paciente ${ }^{(16)}$.

Ideia Central 3: Dificuldades relacionadas ao paciente e ao estilo de vida

[...] a dificuldade é vim para o hospital pra poder pegar
o medicamento, alimentação é muito pouca, então a gente
que faz esse tratamento tem que ter uma boa alimentação
pra que não venha às defesas da gente ficar muito baixa,
precisa dormir tranquilo. Então são essas dificuldades
que a gente tem pra seguir o tratamento [...].

Emerge, a partir dos relatos dos sujeitos, que os fatores relacionados ao paciente e ao seu estilo de vida correlacionam-se com o seu perfil socioeconômico-cultural, e que a experiência anterior de tratamento é uma variável comportamental importante, que pode ser determinada por contextos permanentes ou pontuais. Pacientes com história de dificuldades ligadas à vivência ao longo do tratamento podem acarretar mudanças de acordo com as dificuldades e experiências do paciente, ocasionando momentos de maior ou menor adesão $\mathrm{o}^{(17)}$.

O estilo de vida de alguns pacientes pode se transformar em um fator determinante para a não-adesão. Se ele for usuário de substâncias ilícitas, este fator surge de forma significava associada à baixa adesão. ${ }^{(18)}$ Os estereótipos associados ao uso de tais substâncias dificultam o atendimento do sujeito na sua singularidade, impedindo, assim, um maior auxílio nas suas dificuldades por parte da equipe de saúde. 
Ideia Central 4: Dificuldades com os regimes terapêuticos e confusão sobre os intervalos entre as doses:

[...] uma das dificuldades que tem é aquela coisa que é muito remédio, aí tem horário de um e do outro horário. Ai, às vezes a pessoa se esquece, aí já vai tomar outro horário que não era que a pessoa tem que tomar, já passa uma hora ou duas horas a mais, às vezes fazia mal que eu tava tomando pra TB, tava tomando aquele vermelho de manhã, aí às 10 horas era aquele branco e de tarde era uns azuis […].

Verificamos que as dificuldades mais frequentes enfrentadas pelos pacientes vivendo com HIV e com co-infecção tuberculose/AIDS não estão relacionadas somente à questão socioeconômica, aos fatores sociais e ao estilo de vida, mas também às dificuldades com os regimes terapêuticos e à confusão quanto aos intervalos entre as doses, que são motivos que interferem de forma significativa na adesão de forma continuada e eficaz ao tratamento da tuberculose.

Mudanças de hábitos devido ao uso do medicamento podem também influenciar negativamente na adesão. Outros aspectos que devem ser considerados, no estudo relacionado à adesão à terapêutica, estão presentes nos casos em que o tratamento afeta a relação com a atividade profissional, como medicamentos que causam sonolência em guardas noturnos, ou em casos em que afetam a vida social, como a indicação da não-utilização de bebidas alcoólicas ${ }^{(16)}$.

O conhecimento sobre a doença e o seu tratamento, bem como possíveis experiências repetidas do paciente com hospitalizações, são fatores de extrema importância no que se refere ao uso adequado dos medicamentos. Outro aspecto relevante é a percepção que o paciente tem da melhora dos sintomas ou da sua ausência, principalmente quando há a ocorrência ou não de dor, o que poderá induzi-lo a suspender o tratamento por acreditar que não há mais necessidade do seu uso. A adesão também pode ser afetada pela existência de uma ou mais doenças concomitantes, podendo ocorrer impacto na cognição do sujeito com a necessidade de maior uso de medicações ou pela soma de sintomas ${ }^{(19)}$.

Os fatores relacionados aos próprios pacientes representam recursos, conhecimentos, atitudes, crenças, percepções e expectativas que o paciente possui para lidar com a situação da doença. Alguns fatores dessa natureza podem afetar negativamente na adesão ao tratamento medicamentoso, como por exemplo: o esquecimento, a falta de motivação, a ansiedade sobre possíveis efeitos adversos, a frustração com a equipe de saúde, a ansiedade com regimes complexos, a quantidade de medicamentos e os fatores emocionais ${ }^{(14)}$.

Ideia Central 5: Consumo de drogas ilícitas como barreira à adesão contínua ao tratamento:

[...] rapaz eu parei algumas vezes porque eu não vou mentir, eu usei drogas, aí fico pra tomar os remédios e a droga, tinha que dizer ou era a droga ou o remédio, o por isso que eu desisti de tomar os remédios, mais graças a Deus voltei a tomar direitinho o remédio e bola pra frente [...].

Consoante aos discursos, podemos inferir que o uso de drogas ilícitas revelou-se como um fator que impossibilita tanto à adesão à terapia antituberculose e ARV como a regularidade desta. Verificamos, a partir dos relatos, que muitas vezes o uso da droga estimula o sujeito à tomada de decisão sob uma percepção negativa em relação à continuidade da terapêutica medicamentosa.

Percebemos que o consumo de drogas e a sua inserção na vida do sujeito, mesmo ele tendo toda a compreensão dos malefícios comprovados pelo uso dessas substâncias, muitas vezes está associado à busca pelo esquecimento das dificuldades enfrentadas na vida e pela superação de sentimentos de frustração, solidão e medo da morte.

Ideia Central 6: Uso recreacional do álcool como fator de interrupção da tomada do remédio:

[...] não tomei porque bebi cerveja..., eu não vou mentir às vezes a pessoa sai e bebe, aí quando vêjá bebeu, então é mesmo que nada tomar remédio..., não, eu não perguntei pro médico se podia..., tenho medo de misturar, eu acabo deixando ele [o medicamento] de lado se eu vou tomar alguma bebida alcoólica[...].

O uso recreacional do álcool foi mencionado com frequência pelos entrevistados como fator que dificulta a adesão continuada ao tratamento terapêutico da tuberculose. Percebemos, nos relatos, diversos episódios de interrupções no uso de medicamentos em virtude do consumo de bebidas alcoólicas nos momentos de lazer.

Diversos estudos destacam o papel do álcool no aumento dos níveis séricos, interferindo no aumento do risco de eventos adversos da terapia 
antituberculose e ARV, como também elevando o perigo de toxicidades e mesmo possível perda de eficácia dos medicamentos.

As causas do alcoolismo podem ser consideradas multifatoriais, associadas a fatores biopsicossociais, que se complementam na explicação etiológica do consumo do álcool. Seu uso abusivo pode acarretar desordens orgânicas, revelando-se como fator que impossibilita tanto a adesão como a regularidade do tratamento terapêutico ${ }^{(20)}$.

\section{CONSIDERAÇÕES FINAIS}

Com os resultados deste estudo foi possível perceber que os pacientes vêm atribuindo somente a eles as dificuldades para a adesão ao tratamento e mencionam alguns fatores que contribuem para a não-adesão ao tratamento. Dentre estes destacam-se: dificuldades em realizar o tratamento; dificuldades relacionadas ao paciente e ao estilo de vida; dificuldades com os regimes terapêuticos e confusão sobre os intervalos entre as doses; consumo de drogas ilícitas como barreira à adesão contínua ao tratamento e uso recreacional do álcool como fator de interrupção de uso do remédio.

Notamos, a partir dos depoimentos dos entrevistados, que o uso do álcool e o consumo de drogas ilícitas são considerados como fatores associados à falta de uma adesão continuada à terapêutica medicamentosa de doenças crônicas como a tuberculose, tendo se apresentado como barreira e desafio para as pessoas que vivem com HIV/AIDS e com a co-infecção tuberculose.

Vale ressaltar que mesmo não tendo o conhecimento científico nem o esclarecimento dos profissionais de saúde, os pacientes se apropriam do senso comum sobre o malefício que existe da associação de álcool e/ou drogas ilícitas com os medicamentos.

A não-adesão ao tratamento medicamentoso, por parte dos pacientes, acontece com todos, tendo os motivos pessoais e os intervalos de tempo caráter variável, o que nos leva a refletir sobre a não-conscientização desses pacientes sobre a importância de cumprir adequadamente o tratamento.

Outros pontos destacados na pesquisa foram: a opinião e as atitudes negativas relacionadas à medicação. O excesso de remédios, o uso da medicação por períodos longos e a necessidade de observar os variados e diferentes momentos de tomar as doses da medicação são apontados como dificuldades enfrentadas pelos pacientes.

As barreiras e os desafios apresentados pelos pacientes, em especial aqueles que estão no início do tratamento, requerem maior envolvimento entre os profissionais dos serviços de saúde e os pacientes, sendo necessários profissionais mais conscientes sobre os aspectos que interferem na adesão ao tratamento e que possuam, ainda, habilidades para proporcionar um atendimento crítico-reflexivo aos pacientes sobre a importância da continuidade do tratamento.

Ressaltamos que a não-adesão, além de afetar as condições clínicas do paciente, afeta também a sua qualidade de vida, agravando a doença e acarretando a necessidade de procedimentos com custos mais elevados e complexos.

\section{REFERÊNCIAS}

1 Ticona AM, Quilca DI. Tuberculosis en pacientes con VIH/SIDA. Acta Méd Peruana. 2008; 25(4): 247-254.

2 Organização Mundial da Saúde. Trabalhando juntos pela saúde. Brasília: Ministério da Saúde; 2007.

3 Nações Unidas. UNAIDS. Relatório global sobre a epidemia de AIDS. Genebra; 2010.

4 Rodrigues JRAL, Ruffino-Netto A, Castilho EA. Distribuição especial da co-infecção M. tuberculosis/ HIV no Estado de São Paulo, 1991-2001. Rev Saúde Pública. 2006; 40 (2):265-70.

5 Ministério da Saúde(BR). Secretaria de Vigilância em Saúde. Guia do Programa de Vigilância Epidemiológica da tuberculose multirresistente (versão preliminar). Rio de Janeiro: Ministério da Saúde; 2009.

6 Ministério da Saúde (BR). Secretaria de Vigilância em Saúde. Informe Eletrônico da Tuberculose. Bol Eletr Epidemiol [Internet]. 2009 [citado 2012 jun 14]; 9(2):1-4. Disponível em: http://portal.saude. gov.br/portal/arquivos/pdf/ano09_no2_inf_eletr_tb.pdf

7 Secretaria da Saúde (CE). Informe Epidemiológico Tuberculose. Fortaleza: Secretaria da Saúde; 2009.

8 Oliveira HB, Marin-León L, Cardoso JC. Perfil de mortalidade de pacientes com tuberculose relacionada à comorbidade tuberculose-Aids. Rev Saúde Pública. 2004; 38(4): 503-10. 
9 Silva JBJR. Tuberculose: guia de vigilância epidemiológica. J Bras Pneumol. 2004; 30(suppl.1):S57-S86.

10 Leopardi MT, Beck CLC, Nietsche EA, Gonzales RMB. Metodologia da Pesquisa na Saúde. Santa Maria: Pallotti; 2001.

11 Lefevre F, Lefevre AMC, Teixeira JJV. O Discurso do Sujeito Coletivo. Uma nova abordagem metodológica em pesquisa qualitativa. Caxias do Sul: Educs; 2000.

12 Lima HMM. Adesão ao tratamento de HIV/AIDS por pacientes com AIDS, Tuberculose e usuários de drogas de São Paulo [tese]. São Paulo: Universidade de São Paulo; 2006.

13 World Health Organization. Adherence to long-term therapies: evidence for action. Geneva, Switzerland: World Health Organization; 2003.

14 Melchior R, Nemes, MIB, Alencar, TMD, Buchalla, CM. Desafios da adesão ao tratamento de pessoas vivendo com HIV/Aids no Brasil. Rev Saúde Pública. 2007; 41(Supl. 2):87-93.
15 Vermeire E, Hearnshaw H, Vanroyen P, Denekens J. Patient adherence do treatment: three decades of research. A comprehensive rewiew. J Clin Pharm Ther 2001; 26(5):331-42.

16 Levy RL, Feld AD. Increasing patient adherence to gastroenterology treatment and prevention regimens. Am J Gastroenterol. 1999; 94(7):1733-42

17 Ministério da Saúde (BR). Recomendações para a terapia anti-retroviral em adultos infectados pelo HIV. $7^{\mathrm{a}}$ ed. Brasília; 2008.

18 Colombrini, MRC, Lopes, MRC, Figueiredo, RM de. A Adesão à terapia antiretroviral para HIV/AIDS. Rev Esc Enferm USP. 2006; 40(4):576-81.

19 Dunbar-Jacob J, Mortimer-Stephens MK. Treatment adherence inchronic disease. J Clin Epidemiol. 2001; 54(Suppl 1):S57-60.

20 Vargas D, Labate, RC. Trabalhar com pacientes alcoolistas: satisfação de enfermeiros de hospital geral. Rev Gaúcha Enferm. 2005; 26(2):252-60.

\author{
Endereço do autor / Dirección del autor / \\ Author's address: \\ Izaildo Tavares Luna \\ Rua Carlos Vasconcelos, 1847, ap. 303 \\ 60115-171, Fortaleza, CE \\ E-mail: izaildo@ufc.br
}

